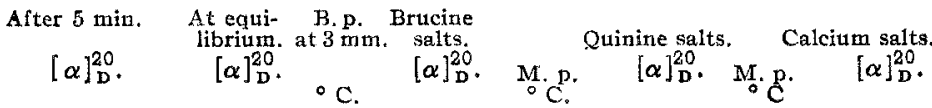

$$
\begin{aligned}
& d \text {-Acid } \quad+20.29^{\circ}+14.97^{\circ} \quad 96 \quad-20.79^{\circ} 169-106.4^{\circ} \quad 149 \quad+17.08
\end{aligned}
$$

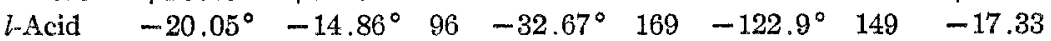

Chicago, Iliminots.

[Contribution hrom the Kent Chemical, Laboratory, University of Chicago.]

\title{
THE PREPARATION OF OPTICALLY-ACTIVE HYDRAZINES. II. THE PREPARATION OF dl-p-SECONDARY-BUTYL- PHENYLHYDRAZINE. THE RESOLUTION OF dl-p-SECONDARY-BUTYL-ANILINE.
}

\author{
By J. W. E. GlattTFid and Edogar Wertemim. ${ }^{3}$
}

Received August 29, 1921.

As stated in the first paper ${ }^{2}$ of this series these attempts to prepare some optically-active phenylhydrazines have been undertaken in order to supply another set of reagents to supplement the alkaloids in the resolution of such $d l$-hydroxy acids as the saccharinic acids. ${ }^{3}$ Furthermore the hope has been entertained that methods of preparation might be found which would make some of the optically-active hydrazines much less expensive than the alkaloids ordinarily used. In the previous attempt only the $d l-p$-trimethylethyl-phenylhydrazine was prepared, such attempts as were made to obtain one of the active forms having failed. In the work reported below it was again found impossible in the time at hand to isolate the active forms of the hydrazine prepared (sec.-butyl-phenylhydrazine) although the hydrazine was obtained in the $d l$ form and the active forms of the corresponding aniline were isolated. In the light of our work up to the present time, it does not seem impossible ultimately to succeed in obtaining some of the active phenylhydrazines and our attempts will therefore continue. In the present paper we report as briefly as seems possible those results of our work that appear to us to be of some general interest or importance.

The plan for the preparation of the optically-active p-sec.-butyl-phenylhydrazines included first the preparation of $d l-s e c$.-butyl-aniline, then the resolution of this compound into the two optical isomers, and finally the preparation from these, of the active phenylhydrazines.

In order to prepare $d l-p$-sec.-butyl-aniline it was necessary first to pre-

1 The dissertation of which this paper is a condensation was presented by $\mathbf{E}$. Werthein as part fulfilment of the requirements for the degree of Doctor of Philosophy in the University of Chicago. The original dissertation is on file in the University library.

2 Glattfeld and Milligan, This Journal, 42, 2322 (1920).

s Glattfeld and Miller, ibid., 42, 2314 (1920). 
pare and in the order named, the following compounds: (1) "methopropenyl benzene" (1-phenyl-1-methyl-2-methyl-ethylene); (2) dl-sec.-butylbenzene; (3) $d l$-p-nitro-sec-butyl-benzene. The first two of these compounds are described in the literature, ${ }^{4}$ and the methods there set forth have been modified somewhat in the unpublished work of R. J. Williams ${ }^{5}$ and A. F. Hardman. ${ }^{6}$ The third compound was first prepared by Williams, ${ }^{5}$ and his work was repeated and extended by Hardman. ${ }^{6}$

In the present paper the preparation of these three compounds will not be described in detail; only departures from published work and from the ordinary course of synthesis will be noted. ${ }^{7}$

$d l-p$-sec.-Butyl-aniline has recently been prepared by Reilly and Hickinbottom $^{8}$ in the course of their investigations in rearrangements of the alkyl-aryl amines. They heated sec.-butyl alcohol, aniline and zinc chloride in an autoclave at a high temperature and isolated this compound, as well as other amines, from the reaction mixture. As they prepared and analyzed several derivatives of the compound, their work will be referred to again.

\section{Experimental Part.}

"Methopropenyl benzene" was prepared by the method of Grignard, from acetophenone, ethyl bromide and magnesium turnings. The acetophenone was added to the thoroughly cooled (with ice and salt) and vigorously stirred ether solution of ethylmagnesium bromide and the impure hydrocarbon was obtained by hydrolysis of the reaction product with ice water and, later, dil. sulfuric acid. The product of the reaction consists in large part of methyl-ethyl-phenyl-carbinol. A fact which does not appear to have been noted before is that most of the carbinol can be changed to the olefine by heating to $100^{\circ}$ for 20 minutes. Fractions which corresponded to the temperatures $t-180^{\circ}, 180-186^{\circ}, 186-191^{\circ}, 191-t^{\circ}$ were taken and dried by means of calcium chloride. Practically the whole of the product after the second fractionation was found in the fraction which had the boiling range 186-191.

The boiling point of the pure compound is variously reported in the literature. The writer's experience agrees with that of Tiffenea $1^{4}$ who gives $186-7^{\circ}$ as the boiling point: at ordinary pressure. For the purposes of this work the entire fraction $186-191^{\circ}$ was used without further treatment for the next step in the synthesis. The best yield of the fraction $186-191^{\circ}$ obtained in 12 runs was $82 \%$; the average yield was $73 \%$. These are calculated from the acetophenone used.

For the preparation of sec.-butyl-benzene, "methopropenyl benzene" was reduced by the use of sodium and moist, but alcohol-free, ether. ${ }^{5}$ Freshly cut sodium was dropped through the condenser and into the hydrocarbon which was dissolved in a layer of ether resting upon water. After $75 \%$ of the sodium had been introduced, the reduction

"Radziszewski, Ber., 9, 260 (1876). Estreicher, Ber., 33, 439 (1900). Klages, Ber., 35, 2641 (1902). Tiffeneau, Ann. chim. phys., [8] 10, 322 (1907).

' Williams, M. S. Thesis of 1918 on file in University of Chicago Library.

" Hardman, M. S. Thesis of 1919 on file in University of Chicago Library.

"Minute details of the procedure followed throughout this work will be found in the complete dissertation of $\mathrm{E}$. Wertheim on file in the University Library, University of Chicago.

Reilly and Hickinbottom, J. Chem. Soc., 117, 120 (1920). 
was interrupted, the ether layer separated from the aqueous layer, and the latter twice extracted with ether and then discarded. The reduction was then resumed as above with a fresh water layer. It was found that removing the alkaline water solution at this stage materially increased the yields. The fraction of boiling range $170-180^{\circ}$ was then obtained from the ether layer and extracts and was used for the next step in the synthesis. Mast of the liquid distilled at $173-4^{\circ}$ which is the boiling point of sec.-butyl-benzene given in the literature. ${ }^{9}$ The best yield obtained in 11 runs was $77 \%$; the average was $67 \%$.

In preparing the $p$-nitro-sec.-butyl-benzene the ordinary course of nitration with nitric and sulfuric acids was followed. The temperature of the reaction mixture was kept between $5^{\circ}$ and $10^{\circ}$. The nitro compound was obtained by steam distillation, ether extraction of the distillate, etc. The fraction which had a boiling range of $130-$ $140^{\circ}$ at $12 \mathrm{~mm}$. was largely $d l-p$-nitro-sec-butyl-benzene. ${ }^{10}$ This fraction was redistiled and the part which boiled at $130^{\circ}$ at $9 \mathrm{~mm}$. was pure $d l$-p-nitro-sec-butylbenzene. The best yield in four runs was $63 \%$; the average was $57 \%$.

The reduction of the nitro compound was carried out in the usual manner with conc. hydrochloric acid and granulated tin. The aniline was obtained from the reaction mixture by the addition of sufficient alkali to make it just alkaline (a large excess of alkali is to be avoided ${ }^{2}$ ), subsequent distillation with steam, extraction of the distillate with ether, etc. The boiling range of the crude compound was $120-130^{\circ}$ at $18 \mathrm{~mm}$. Redistillation yielded pure aniline which boiled at $112^{\circ}$ at $11 \mathrm{~mm}$. The boiling point at atmospheric pressure is given by Reilly and Hickinbottom ${ }^{8}$ as $238^{\circ}$. The acetyl derivative was prepared by the use of acetyl chloride. It was recrystallized from a mixture of water and $95 \%$ alcohol and was found to have a melting point of $125-6^{\circ}$. This is the melting point reported by Reilly and Hickinbottom. ${ }^{8}$

The $d l$-pusec.-butyl-aniline was analyzed in this laboratory by $\mathrm{Mr}$. Williams. ${ }^{5}$ The following results are quoted from his paper.

Analyses. Subs., 0.1437, 0.2311: $\mathrm{CO}_{2}, 0.4241,0.6843 ; \mathrm{H}_{2} \mathrm{O}, 0.1276,0.2046$. Subs., $0.2938,0.2994: \mathrm{N}_{2}, 24.65 \mathrm{cc}$. $\left(20^{\circ}\right.$ and $751 \mathrm{~mm}$ ), $24.1 \mathrm{cc} .\left(20^{\circ}\right.$ and $749 \mathrm{~mm}$ ). Calc. for $\mathrm{C}_{10} \mathrm{H}_{15} \mathrm{~N}$ : C, $80.54 ; \mathrm{H}, 10.07 ; \mathrm{N}, 9.39$. Found: C, 80.50, 80.75; $\mathrm{H}$, $9.94,9.90 ; \mathrm{N}, 9.52,9.11$.

The preparation of $p$-sec.-butyl-aniline was also attempted by the Friedel and Crafts reaction, using acetanilide, sec-butyl chloride, and aluminum chloride. The reaction seemed to go quite smoothly and the desired aniline was obtained by hydrolysis, etc, but in small quantity; the largest yield realized was $14 \%$. The aniline had the proper boiling point, and the acetyl derivative had the proper melting point. When mixed with a sample of the acetyl derivative from the aniline made from acetophenone above, there was no depression of the melting point.

\section{Resolution of $d l$-p-sec.-Butyl-aniline.}

Oxymethylene-camphor and bromo-camphor sulfonic acid were first tried as resolving agents for $d l$-p-sec-butyl-aniline, but with only partial success. Camphor sulfonic acid was found to be a much better reagent. A typical experiment is described below.

Levo-p-sec.-butyl-aniline.-Ten $\mathrm{g}$. of the $d l$-p-sec--butyl-aniline was placed in a beaker and $10 \mathrm{cc}$. of $30 \%$ acetic acid added. To this m xture was added $17 \mathrm{~g} .(15.5$

${ }^{9}$ Klages, Ber., 35, 3509 (1902).

${ }^{10}$ The position of the nitro group as para was established in the work of R. J. Williams to which reference has been made. He oxidized the nitro compound with chromic acid to $p$-nitrobenzoic acid, and proved the identity of the latter substance. 
g. required theoretically) of camphor sulfonic acid as a fine powder. The mixture was stirred until the acid had dissolved. This took place readily and with the production of a small amount of heat. The beaker was then kept on an electric hot-plate at $100^{\circ}$ for 2 hours, or until a considerable part of the acetic acid had evaporated, and the residue was quite thick.

The mass was then allowed to cool, after which $t$ was broken up with a pestle and twice well-washed by decantation with ice-water. The water which adhered was removed as quickly as possible by the use of a Büchner funnel. The moist compound was pressed between filter papers, and then placed in a vacuum desiccator over sulfuric acid. It was generally quite dry in 5 or 6 days. By this treatment the racemate obta ned was fairly pure as shown by the fol owing rotations. These rotations are from 5 different preparations of the racemate. ${ }^{11}$

Rotations. Subs., $0.9862,0.9121,0.5522,0.2262,0.2392 . \quad[\alpha]_{\mathrm{D}}=+25.98$, $25.77,26.11,25.97,26.23$.

The melting point of the dry racemate was $119-120^{\circ}$; it was a light pinkish-yellow powder, and weighed between 17 and $20 \mathrm{~g}$.

The racemic compound was next treated with 50 to $75 \mathrm{cc}$. of pure ether, and allowed to stand with frequent stirring, for about 2 hours. The supernatant liquid changed in this time to a pink color, and the powder became pure white. The mixture was then subjected to filtration by suction, and the white crystals were washed on the filter with dry ether, and then air-dried. The crystals were recrystallized from a mixture of about 5 parts of ethyl acetate to 1 part of acetone, and then had a melting point of $173-4^{\circ}$. A separation had therefore evidently been effected. The sample at this point usually weighed from 5 to $7 \mathrm{~g}$. Rotations in chloroform showed no change after several crystallizations of the same crop from the solvents named above; the value was invariably neat to +32.00 . The following figures show the results of one such experiment.12

$\begin{array}{cccc}\text { Crop. } & \text { Weight. } & \text { subs. } & \\ 1 & G . & \text { G. } & {[\alpha]_{\mathcal{D} .}} \\ 2 & 6.0 & 0.2740 & +31.95 \\ 3 & 3.8 & 0.2282 & +32.32 \\ 4 & 2.2 & 0.3038 & +31.68 \\ & 0.93 & 0.2305 & +31.62\end{array}$

The following figures for a number of samples show the constancy of the rotation. after the first crystallization. These are the rotations of 6 different preparations after the ether treatment of the racemate, and one recrystallization from acetoneethyl acetate mixture of the crystals insoluble in ether.

Rotations. Subs, $0.3329,0.6062,0.6050,0.8351,0.4915,0.5539 \quad[\alpha]_{D}=$ $+31.47,31.22,31.76,31.43,31.54,31.41$.

From these figures it seems fairly certain that one of the pure components has a specific rotation between +31.5 and 32.00 . The free aniline was obtained by treating the camphor sulfonic derivative of this specific rotation first with conc. hydrochloric acid, and treating the crystalline hydrochloride obtained with alkali, etc. The aniline was found to have a light yellow color and to boil at $116.5-118^{\circ}$ at $15 \mathrm{~mm}$. pressure. A

${ }^{11}$ All the rotations in this work were made, unless otherwise stated, in chloroform solution, in a $2 \mathrm{dcm}$. tube and at room temperature. The solution was in each case made up to a volume of $25 \mathrm{cc}$. and quickly filtered, after which the readings were taken with the minimum loss of time.

12 The 2 nd crop $(3.8 \mathrm{~g}$.) was obtained by recrystallization of the 1st crop $(6.0 \mathrm{~g})$. The $3 \mathrm{rd}$ crop ( $2.2 \mathrm{~g}$.) was obtained by recrystallization of the 2 nd crop ( $3.8 \mathrm{~g}$.), etc. 
sample of this aniline when examined in a $0.5 \mathrm{dcm}$. tube gave $\alpha=-0.26$. Analysis of samples gave the following results.

Subs., $0.2986,0.3022: \mathrm{CO}_{2}, 0.8832,0.8932 ; \mathrm{H}_{2} \mathrm{O}, 0.2724,0.2796$. Subs., 0.3386 , $0.3422: \mathrm{N}_{2}, 29.4 \mathrm{cc}$. $\left(24^{\circ}\right.$ and $740.5 \mathrm{~mm}$ ), $29.5 \mathrm{cc}$. $\left(24.5^{\circ}\right.$ and $748 \mathrm{~mm}$.). Calc. for $\mathrm{C}_{10} \mathrm{H}_{15} \mathrm{~N}$ : C, $80.54 ; \mathrm{H}, 10.07 ; \mathrm{N} .9 .39$. Found: C, 80.67, 80.61; H, 10.14 $1023 ; \mathrm{N}, 9.49,9.46$.

The acetyl derivative was made by treating the aniline with acetyl chloride, and the resulting crystals were recrystallized from a mixture of water and $95 \%$ alcohol. The pure white crystals were found to have a melting point of $123-4^{\circ}$. When the rotation of a sample of $0.5376 \mathrm{~g}$. was taken in chloroform solution, the specific rotation was found to be $[\alpha]_{D}^{25}=+0.9301$.

Dextro-p-sec-Butyl-aniline.-The dextro-rotatory aniline was obtained from the soluble camphor sulfonic acid derivative left in the filtrates after the crystalline derivative had been removed (see above). This soluble compound remained as a dark-brown or black semi-solid gum after all of the solvent had been removed by evaporation. Solutions were too dark for determination of the rotation.

The gum was first dissolved in sodium-dried ether and separated by filtration from a few crystals of the less soluble isomer which it contained. The ether was next removed by evaporation, and the gum covered with conc. hydrochloric acid and heated to $100^{\circ}$ for 5 hours. Excess of sodium hydroxide was then added, and the aniline recovered as ustal. The boiling point of the aniline was $123-5^{\circ}$ at $20 \mathrm{~mm}$. Part of this sample was placed in a $0.5 \mathrm{dcm}$. tube and gave $\alpha=+0.26$. This rotation is equal and opposite to that of the aniline from the crystalline camphor sulfonic compourid, and indicates that the sample was probabiy pure.

An attempt to convert $9.6 \mathrm{~g}$. of the $l$-sec.-butyl-aniline into the active phenylhydrazine failed entirely although the procedure under which the $d l$-aniline had been successfully converted into the $d l$-hydrazine (see below) was followed carefully. Furthermore, these conditions failed also in one instance in the making of the $d l$-hydrazine. No reason for these failures can be offered at the present time, but it is hoped that perfect conditions for the conversion will ultimately be found.

\section{Preparation of $d l-p-s e c$. -Butyl-phenylhydrazine.}

This compound was prepared from the corresponding aniline as follows.

The aniline was first treated with conc. hydrochloric acid and diazotized. The addition of the nitrite solution took some time as it was found to be important to keep the temperature of the reaction mixture always below $0^{\circ}$. The diazonium salt solution was poured at once into a cold solution of sodium sulfite, giving a rich red solution, a sample of which was only slightly cloudy after having been boiled for one minute. This solution was then reduced in the usual way by the Fischer method ${ }^{13}$ with zinc dust and acetic acid. The reaction mixture was heated nearly to the boiling point during the reduction in order to make sure of completeness of reduction. From the cooled solution the solid sulfonate was separated by filtration.

The $d l$-p-sec.-butyl-phenylhydrazine sodium sulfonate crystallizes from a water solution in beautiful white plates with a pearly lustre. These are stable in the air for about 2 hours when they darken slightly. After one crystallization from $95 \%$ alcohol the crystals were well dried in a vacuum desiccator over sulfuric acid.

${ }^{13}$ Fis 
Analyses. Subs, $0.1743,0.1626: \mathrm{N}_{2}, 15.4 \mathrm{cc}$. $\left(19^{\circ}\right.$ and $\left.753.4 \mathrm{~mm}.\right), 14.8 \mathrm{cc}$. (21.3 $3^{\circ}$ and $754.8 \mathrm{~mm}$.).

Subs., 1.0948, 1.3103: $\mathrm{BaSO}_{4}, 0.9557,1.1544$. Calc. for $\mathrm{C}_{10} \mathrm{H}_{15} \mathrm{~N}_{2} \mathrm{SO}_{3} \mathrm{Na}: \mathrm{N}$, $10.53 ; \mathrm{S}, 12.03$. Found: $\mathrm{N}, 10.11,10.36 ; \mathrm{S}, 11.99,12.10$.

To obtain the hydrochloride of the hydrazine, the sulfonate was covered with conc. hydrochloric acid, and the mixture heated to boiling for 8 hours, during which time fresh acid was supplied as needed. The solid hydrochloride was obtained as a mass of white crystals from the cooled solution. These were treated with sodium hydroxide, etc, to give the hydrazine. The crude hydrazine was subjected to distillation at reduced pressure in an atmosphere of hydrogen and boiled at $145-158^{\circ}$ at $16 \mathrm{~mm}$. When redistilled it had a boiling range of $155-7^{\circ}$ at $18 \mathrm{~mm}$.

The $d l$-p-sec.-butyl-phenylhydrazine is a thick light-yellow oil when first distilled but soon turns to a maroon color in the air. In sealed tubes it keeps its light yellow color indefinitely.

Analyses. Subs., $0.2390,0.2023: \mathrm{N}_{2}, 36.5 \mathrm{cc} .\left(22.0^{\circ}\right.$ and $\left.738.5 \mathrm{~mm}.\right), 31.4$ ce. $\left(25.8^{\circ}\right.$ and $745 \mathrm{~mm}$. $)$.

Calc. for $\mathrm{C}_{10} \mathrm{H}_{10} \mathrm{~N}_{2}: \mathrm{N}, 17.08$. Found: $16.85,16.99$.

Only a small amount of the $d l$-hydrazine was available for a study of derivatives and consequently only a few preliminary tests could be made. It seems to form only gummy derivatives with $d$-glucose and $l$-xylose. With $d$-galactose it apparently forms a crystalline derivative with a melting point of $152-155^{\circ}$. With $l$-arabonic and $d$-gluconic lactone no crystalline derivative could be obtained but there was indication of the formation of a crystalline derivative with $d$-mannonic lactone.

\section{Summary.}

This paper describes the preparation and some of the properties of $d l$-p-sec.-butyl-phenylhydrazine; the preparation of $d l$-p-sec.-butyl-aniline, and the resolution of the latter into the $d$ and $l$ components. The following physical constants are given.

\begin{tabular}{|c|c|c|c|}
\hline & $\begin{array}{l}\text { Boiling point. } \\
{ }^{\circ} \mathrm{C} .\end{array}$ & $\begin{array}{c}\text { Pressure. } \\
\text { Mm. }\end{array}$ & $\begin{array}{c}\alpha \text { in } \\
0.5 \mathrm{dcm} \text {. tube. }\end{array}$ \\
\hline$d l$-Aniline & 112 & 11 & \\
\hline d-Aniline. . & $123-5$ & 20 & +0.26 \\
\hline$l$-Aniline. & $116.5-118$ & 15 & -0.26 \\
\hline$d l$-Hydrazine. . . . . . . . . . . . & $155-7$ & 18 & \\
\hline$d l$-Aniline acetyl derivative. . . . . & $125-6$ & & \\
\hline$l$-Aniline acetyl derivative.... & $123-4$ & ]$_{D}=+$ & +0.9301 \\
\hline
\end{tabular}

Chrcago, Ilfinots. 